\title{
A DIFFERENCE BETWEEN MINIMAL AND ORDINARY FINE TOPOLOGY IN FUNCTION THEORY
}

\author{
J. S. HWANG ${ }^{1}$
}

\begin{abstract}
After Choquet (see Brelot and Doob [6, p. 404]), we recently have presented an alternative answer of Doob's problem [8] by showing that there is a Blaschke product $B$ having the minimal fine cluster value 0 and the angular limit 1 at $\infty$. In our construction, the zeros of $B$ lie on both the first and fourth quadrant. Naturally, we may ask if a product having the same property, but having zeros which lie on only one quadrant, can be constructed. We show that the answer to this question is no for the minimal fine topology, but yes for the ordinary one. There is a significant difference between these two topologies in function theory.
\end{abstract}

1. Introduction. Let $H=\{z: \operatorname{Re}>0\}$ be the right half-plane and let $\partial H$ be the boundary of $H$. Then the Martin boundary [16] is simply the union $\partial H \cup\{\infty\}$. Throughout this paper, we shall be devoted to the study of thin set at $\infty$. Following Brelot [2], we say that a set $S \subset H$ is ordinarily thin at $\infty$ if and only if there is a positive superharmonic function $u(z)$ in some neighborhood of $\infty$ such that

$$
u(\infty)<\infty \text { and } \lim _{z \rightarrow \infty} u(z)=\infty, \quad \text { where } z \in S .
$$

Also following Brelot [5] or Lelong [15], we say that a set $S \subset H$ is minimally thin at $\infty$ if and only if the reduced function

$$
R_{x}^{S}(z) \not \equiv x, \quad \text { where } z=x+i y \text { in } H .
$$

The basic properties of these two topologies can be found in Brelot [5]. With respect to these two topologies, we say that (see Brelot and Doob [6]) a function $f(z)$ has an ordinary (or minimal) fine cluster value $v$ at $\infty$, if there is a set $S \subset H$ which is not ordinarily (or minimally) thin at $\infty$ such that $\lim _{z \rightarrow \infty} f(z)=v$, where $z \in S$. As usual (see Collingwood and Lohwater [7, p. 73]), a function $f$ has an angular cluster value $v$ at $\infty$, if there is a Stolz angle of the form

$$
\Delta_{\epsilon}=\{z:|\arg z| \leq \pi / 2-\epsilon, 0<a \leq|z|<\infty\}
$$

and a sequence of points $P_{n} \in \Delta_{\epsilon}$ such that

$$
\lim _{n \rightarrow \infty} P_{n}=\infty \text { and } \lim _{n \rightarrow \infty} f\left(P_{n}\right)=v .
$$

Received by the editors March 25, 1980 and, in revised form, July 31, 1981.

1980 Mathematics Subject Classification. Primary 31A15; Secondary 30D50.

Key words and phrases. Fine topology, cluster value, Blaschke product.

${ }^{1}$ I am indebted to the Referee for his many valuable comments and corrections, and in particular his contribution of Lemma 1 which improves much of my original Theorem 1 and answers a question of mine in the last section 
We say that $f$ has angular limit $v$ if the above two equalities hold for any sequence of points in $\Delta_{\epsilon}$.

In [8], Doob proved that if $f$ is a function normal in a disk in the sense of Lehto and Virtanen [14], then every angular cluster value at a boundary point is also a minimal fine cluster value at the point. He then asked whether or not the converse of this theorem is true. Recently, we have answered this question in the negative sense [12]. In fact, we have constructed a Blaschke product $B$ which has the minimal fine cluster value 0 and the angular limit 1 at $\infty$.

In our construction, the zeros of $B$ lie on both the first and fourth quadrant. Naturally we may ask if a product having the same property, but having zeros which lie on only one quadrant, can be constructed. We claim that the answer to this question is no for minimal fine topology, but yes for the ordinary one. There is a significant difference between these two topologies in function theory. Of course, in potential theory the difference between these two topologies has been studied by many authors. (See, for instance, Brelot [3, 4], Lelong [15], Naïm [17], Jackson [13], Essén and Jackson $[9,10]$.)

Recall that a Blaschke product $B\left(z,\left\{z_{n}\right\}\right)$ associated with a sequence $\left\{z_{n}\right\}$ is defined by (see Hille $[11$, p. 457])

$$
B\left(z,\left\{z_{n}\right\}\right)=\prod_{n=1}^{\infty} \frac{z_{n}-z}{\bar{z}_{n}+z} \frac{\bar{z}_{n}}{z_{n}}, \quad \text { where } \sum_{n=1}^{\infty} \frac{\operatorname{Re} z_{n}}{1+\left|z_{n}\right|^{2}}<\infty .
$$

With the help of the above notions, we are now able to describe a difference between minimal and ordinary fine topology as follows.

THEOREM 1. Let $B\left(z,\left\{z_{n}\right\}\right)$ be the Blaschke product defined as in (1). If the sequence $\left\{z_{n}\right\}$ lies in the first quadrant and $\lim _{x \rightarrow \infty} B\left(x,\left\{z_{n}\right\}\right)=v \neq 0$, then the value 0 is not a minimal fine cluster value of $B$ at $\infty$.

THEOREM 2. There does exist a sequence $\left\{z_{n}\right\}$ lying in the first quadrant such that the associated Blaschke product satisfies $\lim _{x \rightarrow \infty} B\left(x,\left\{z_{n}\right\}\right)=v \neq 0$ and the value 0 is an ordinary fine cluster value of $B$ at $\infty$.

2. Minimal thin sets. Following Lelong-Ferrand [15], we let $\lambda_{S}$ be the fundamental distribution of a bounded set $S \subset H$ such that the Green potential

$$
\hat{R}_{x}^{S}(z)=\int_{\partial S} \log \left|\frac{w+\bar{z}}{w-z}\right| d \lambda_{S}(w)=x, \quad \text { q.e. for } z=x+i y \in S,
$$

and define the Green energy of $\lambda_{S}$ by

$$
\gamma(S)=\int_{\partial S} \hat{R}_{x}^{S}(z) d \lambda_{S}(z)
$$

We say that a set $E \subset H$ is minimally thin at $\infty$ iff

$$
\sum_{n=1}^{\infty} 2^{-2 n} \gamma\left(E_{n}\right)<\infty
$$

where $E_{n}=E \cap\left\{z: 2^{n} \leq|z|<2^{n+1}\right\}$.

To prove Theorem 1 , we shall need the following result which was suggested and proved by the Referee: 
LEMMA 1. Let $B(z)=B\left(z,\left\{z_{n}\right\}\right)$ be as in (1). If the sequence $\left\{z_{n}\right\}$ satisfies

$$
\sum_{n=1}^{\infty} x_{n}\left|z_{n}\right|^{-1}<\infty, \quad \text { where } x_{n}=\operatorname{Re} z_{n}
$$

then for each $\epsilon, 0<\epsilon<1$, the set $S=\{z:|B(z)| \leq \epsilon\}$ is minimally thin at $\infty$, so that the value 0 is not a minimal fine cluster value of $B$ at $\infty$.

ProOF. Let $d \nu$ be the measure with point masses at the zeros of $B$, then

$$
\log \frac{1}{|B(z)|}=\int \log \left|\frac{z+\varsigma}{z-\varsigma}\right| d \nu(\varsigma)
$$

and

$$
p_{n}(z)=\left(2^{n+1} \log \frac{1}{|B(z)|}\right) / \log \frac{1}{\epsilon} \geq \operatorname{Re}
$$

on $E_{n}=S \cap\left\{z: 2^{n} \leq|z|<2^{n+1}\right\}$.

Let $\operatorname{Re}=x$ and $\operatorname{Re} \zeta=\xi$, then the Green function

$$
g(z, \varsigma)=\log \left|\frac{z+\bar{\zeta}}{z-\varsigma}\right|=\frac{1}{2} \log \left[1+\frac{4 x \xi}{|z-\varsigma|^{2}}\right] \leq \frac{2 x \xi}{|z-\varsigma|^{2}} .
$$

Clearly, for $2^{n} \leq|z|<2^{n+1}$ and $|\zeta| \geq 2^{n+2}$, we have

$$
\frac{\xi}{|z-\varsigma|^{2}} \leq \frac{\xi}{(|\zeta|-|z|)(|\zeta|(1-|z / \zeta|))} \leq \frac{1}{2^{n}} \frac{\xi}{|\zeta|}
$$

It follows from (5), (8), and (9) that there is an integer $N=N(\epsilon)$ such that $n \geq N$ implies

$$
\begin{aligned}
\left(2^{n+1} / \log \frac{1}{\epsilon}\right) & \int_{|\zeta| \geq 2^{n+2}} g(z, \varsigma) d \nu(\varsigma) \\
& \leq\left(4 / \log \frac{1}{\epsilon}\right) x \int_{|\zeta| \geq 2^{n+2}} \frac{\xi}{|\zeta|} d \nu(\varsigma) \leq \frac{x}{4}
\end{aligned}
$$

By the same argument, we have for $2^{n} \leq|z|<2^{n+1}$ and $|\zeta| \leq 2^{n} \delta$, where $0<\delta=\delta(\epsilon)$ is sufficiently small,

$$
\begin{aligned}
\left(2^{n+1} / \log \frac{1}{\epsilon}\right) & \int_{|\varsigma| \leq 2^{n} \delta} g(z, \varsigma) d \nu(\varsigma) \\
& \leq\left(5 / \log \frac{1}{\epsilon}\right) x \delta \int_{|\varsigma| \leq 2^{n} \delta} \frac{\xi}{|\zeta|} d \nu(\varsigma) \leq \frac{x}{4} .
\end{aligned}
$$

Combining (6), (7), (10), and (11), we obtain

$$
x \leq\left(2^{n+2} / \log \frac{1}{\epsilon}\right) \int_{2^{n} \delta<|\varsigma|<2^{n+2}} g(z, \varsigma) d \nu(\varsigma) .
$$

Now, let $\lambda_{E_{n}}$ be the fundamental distribution of the set $E_{n}$. Then by (2), (3), and (12), we find that

$$
\begin{aligned}
\gamma\left(E_{n}\right)=\int_{\partial E_{n}} x d \lambda_{E_{n}} & \leq\left(2^{n+2} / \log \frac{1}{\epsilon}\right) \int_{2^{n} \delta<|\zeta|<2^{n+2}}\left[\int_{\partial E_{n}} g(z, \zeta) d \lambda_{E_{n}}\right] d \nu(\varsigma) \\
& \leq\left(2^{n+2} / \log \frac{1}{\epsilon}\right) \int_{2^{n} \delta<|\varsigma|<2^{n+2}} \xi d \nu(\varsigma) .
\end{aligned}
$$


This together with (5) yields that

$$
\sum_{n=N}^{\infty} 2^{-2 n} \gamma\left(E_{n}\right) \leq\left(16 / \log \frac{1}{\epsilon}\right) \sum_{n=N}^{\infty} \int_{2^{n} \delta<|\zeta|<2^{n+2}} \frac{\xi}{|\zeta|} d \nu(\zeta)<\infty .
$$

Hence the set $S$ is minimally thin at $\infty$ due to (4).

3. Proof of Theorem 1. In view of (1), we write $B(z)=B\left(z,\left\{z_{n}\right\}\right)$ and denote the argument of its factors by

$$
\theta_{n}(x)=\arg \frac{z_{n}-x}{\bar{z}_{n}+x} \frac{\bar{z}_{n}}{z_{n}}, \quad n=1,2, \ldots
$$

Then we have

$$
\phi(x)=\arg B(x)=\sum_{n=1}^{\infty} \theta_{n}(x) .
$$

We shall prove that the series on the right-hand side of (14) converges absolutely and uniformly. For this, we let $z_{n}=x_{n}+i y_{n},\left(z_{n}-x\right) \bar{z}_{n}=\left|\left(z_{n}-x\right) \bar{z}_{n}\right| e^{i \alpha}$, and $\left(\bar{z}_{n}+x\right) z_{n}=\left|\left(\bar{z}_{n}+x\right) z_{n}\right| e^{i \beta}$. Then by a simple computation we obtain

$$
\sin \theta_{n}(x)=\sin (\alpha-\beta)=\frac{2 x^{2} x_{n} y_{n}}{\left|z_{n}\right|^{2}\left|\left(z_{n}-x\right)\left(\bar{z}_{n}+x\right)\right|} .
$$

Since all $z_{n}$ lie on the first quadrant, i.e. $x_{n}>0, y_{n}>0$, it follows from (15) that all $\theta_{n}(x)>0$. This together with the hypothesis $B(x) \rightarrow v$, as $x \rightarrow \infty$, concludes the absolute convergence of (14).

To prove the uniform convergence of (14), we shall need a theorem of Lindelöf [7, Theorem 2.3] which shows us that the radial limit $v$ of $B$ is also the angular limit of $B$ at $\infty$. Since $v \neq 0$, it follows that this sequence $\left\{z_{n}\right\}$ tends to infinity tangentially. This in turn implies that for some $a>0$ and some integer $N>0$,

$$
y_{n}>a x_{n}, \quad n=N, N+1, \ldots
$$

We then obtain the following two inequalities:

$$
\begin{gathered}
\frac{x^{2}}{\left|\left(z_{n}-x\right)\left(\bar{z}_{n}+x\right)\right|}<\frac{\sqrt{1+a^{2}}}{2}, \\
\frac{2 y_{n}}{\left|z_{n}\right|}>\frac{2 a}{\sqrt{1+a^{2}}}, \quad n=N, N+1, \ldots
\end{gathered}
$$

Owing to (15) and (16), we conclude the uniform convergence of the series in (14). It then follows from Fatou's lemma that

$$
\begin{aligned}
\arg v & =\lim _{x \rightarrow \infty} \phi(x)=\lim _{x \rightarrow \infty} \sum_{n=1}^{\infty} \theta_{n}(x) \geq \sum_{n=1}^{\infty} \lim _{x \rightarrow \infty} \theta_{n}(x) \\
& =\sum_{n=1}^{\infty} \sin ^{-1} \frac{2 x_{n} y_{n}}{\left|z_{n}\right|^{2}} \geq \sum_{n=1}^{\infty} \frac{2 x_{n} y_{n}}{\left|z_{n}\right|^{2}}
\end{aligned}
$$

Combining (17) and (18), we obtain

$$
\sum_{n=1}^{\infty} \frac{x_{n}}{\left|z_{n}\right|}<\frac{\sqrt{1+a^{2}}}{2 a} \sum_{n=1}^{\infty} \frac{2 x_{n} y_{n}}{\left|z_{n}\right|^{2}}<\infty
$$


This shows that the condition of Lemma 1 is true and therefore the value 0 cannot be a minimal fine cluster value of $B$ at $\infty$.

4. Ordinary thin sets. To prove Theorem 2, we shall need the following Wiener criterion [18] of thin sets in the ordinary sense (see Brelot [2, p. 325] and Essén and Jackson [9, Theorem 7]):

LEMMA 2. Let $\left\{z_{n}\right\}$ be a sequence of points satisfying $c^{n} \leq\left|z_{n}\right|<c^{n+1}$, for $n=1,2, \ldots$, and let $D_{n}=\left\{z:\left|z-z_{n}\right|<r_{n}\right\}$. Then the union $S=\bigcup_{n=1}^{\infty} D_{n}$ is thin at 0 or $\infty$ depending on $c<1$ or $c>1$ respectively, if and only if

$$
\sum_{n=1}^{\infty} n\left\{\log \left(\left|z_{n}\right| / r_{n}\right)\right\}^{-1}<\infty
$$

5. Hyperbolic metric. To prove Theorem 2, we shall also need the notion of hyperbolic metric. As usual (see Hille [11, p. 241]), the hyperbolic metric between two points $z, w \in H$ is defined by

$$
d(z, w)=\frac{1}{2} \log \frac{|\bar{z}+w|+|z-w|}{|\bar{z}+w|-|z-w|} .
$$

Since the hyperbolic metric is conformally invariant and a bounded function is normal, by a result of Bagemihl and Seidel [1, Lemma 1], we immediately obtain the following:

LEMMA 3. If $f(z)$ is a function bounded and analytic in $H$ and if $\left\{z_{n}\right\}$ and $\left\{w_{n}\right\}$ are two sequences of points in $H$ for which $d\left(z_{n}, w_{n}\right) \rightarrow 0$, as $n \rightarrow \infty$, then the existence of one limit, say $\lim _{n \rightarrow \infty} f\left(z_{n}\right)=c$, implies the other $\lim _{n \rightarrow \infty} f\left(w_{n}\right)=c$.

6. Proof of Theorem 2. For simplicity, we choose

$$
z_{n}=1+i 2^{n} \quad \text { and } \quad r_{n}=2^{-n}, \quad n=1,2, \ldots
$$

We shall first prove that the set $S=\bigcup_{n=1}^{\infty} D_{r_{n}}\left(z_{n}\right)$, is not ordinarily thin at $\infty$. In view of (19), we have

$$
\sum_{n=1}^{\infty} n\left\{\log \left(\left|z_{n}\right| / r_{n}\right)\right\}^{-1}>\sum_{n=1}^{\infty} \frac{n}{2 n \log 2}=\infty .
$$

It follows from Lemma 2 that the set $S$ is not ordinarily thin at $\infty$.

Next, we shall show that

$$
\lim _{z \rightarrow \infty} B(z)=0, \quad \text { where } z \in S .
$$

To prove (21), we let $w_{n}$ be an arbitrary point in $D_{r_{n}}\left(z_{n}\right)$. Then clearly we have $\left|z_{n}-w_{n}\right| \leq 2^{-n}$ and $\left|\bar{z}_{n}+w_{n}\right|>1$. It follows easily from (20) that $\lim _{n \rightarrow \infty} d\left(z_{n}, w_{n}\right)=0$. Since the product $B$ is analytic and bounded by one in $H$, by Lemma 3 we conclude the assertion (21) so that the value 0 is an ordinary fine cluster value of $B$ at $\infty$.

Finally, it is easy to show that

$$
\lim _{x \rightarrow \infty} B(x)=v \neq 0, \quad \text { where } x>0 .
$$

This completes the proof.

7. Remark. In Theorem 2, we may ask whether there can be constructed a Blaschke product for which the limit $v$ has absolute value less than one. It was answered by the Referee that such a construction is impossible. 


\section{REFERENCES}

1. F. Bagemihl and W. Seidel, Sequential and continuous limits of meromorphic functions, Ann. Acad. Sci. Fenn. Ser. A I Math. 280 (1960).

2. M. Brelot, Points irréguliers et transformations continues en théorie du potentiel, J. Math. Pures Appl. 19 (1940), 319-337.

3. 155-168.

4. - Aspect statistique et comparé des deux types d'effilement, An. Acad. Brasil. Ciênc. 37 (1965), 1-15.

5. __ Axiomatique des fonctions harmoniques, Les Presses de l'Université de Montréal, 1966.

6. M. Brelot and J. L. Doob, Limites angulaires et limite fines, Ann. Inst. Fourier (Grenoble) 13 (1963), 395-415.

7. E. F. Collingwood and A. J. Lohwater, The theory of cluster sets, Cambridge Univ. Press, London and New York, 1966.

8. J. L. Doob, Conformally invariant cluster value theory, minois J. Math. 5 (1961), 521-549.

9. M. Essén and H. L. Jackson, A comparison between thin sets and generalized Azarin sets, Canad. Math. Bull. 18 (1975), 335-346.

10. _ $\ldots$ On the covering properties of certain exceptional sets in a half space, Hiroshima Math. J. 10 (1980), 233-262.

11. E. Hille, Analytic function theory. II, Ginn, Boston, Mass., 1962.

12. J. S. Hwang, On a problem of Doob about the fine topology and normal functions, Proc. Amer. Math. Soc. 82 (1981), 239-242.

13. H. L. Jackson, Some results on thin sets in a half plane, Ann. Inst. Fourier (Grenoble) 20 (1970), 201-218.

14. O. Lehto and K. I. Virtanen, Boundary behaviour and normal meromorphic functions, Acta Math. 97 (1957), 47-65.

15. J. Lelong-Ferrand, Etude au voisinage de la frontiere des fonctions surharmoniques positives dans un demi-espace, Ann. Sci. École Norm. Sup. (4) 66 (1949), 125-159.

16. R. S. Martin, Minimal positive harmonic functions, Trans. Amer. Math. Soc. 49 (1941), 127172.

17. L. Naïm, Sur le rôle de la frontière de R. S. Martin dans la théorie du potentiel, Ann. Inst. Fourier (Grenoble) 7 (1957), 183-285.

18. N. Wiener, Certain notions in potential theory, J. Math. Phys. Mass. Inst. Tech. 3 (1924), 24-51.

\section{Institute of Mathematics, ACademia Sinica, Taipei, Taiwan}

